\title{
Specificity of New Diagnostic Criteria for Left Ventricular Hypertrophy
}

\author{
Elaine Clark, Peter W Macfarlane \\ University of Glasgow, Scotland
}

\begin{abstract}
The aim of this study was to assess the recent publication of new Peguero/Lo Presti (P-LP) ECG criteria for left ventricular hypertrophy (LVH) which purported to have a higher sensitivity than commonly used existing criteria but with no significant loss of specificity $(\geq 90 \%)$ compared to the other $L V H$ criteria..

ECGs from 1496 apparently healthy adults from the Glasgow normals ECG database were used to assess specificity. The new P-LP criteria (maximum $S$ amplitude in any lead plus $S$ amplitude in V4) were assessed together with the long established Sokolow-Lyon and Cornell criteria (the latter being sex based) for LVH. The Glasgow criteria were also assessed.

The specificity of the new P-LP criteria were found to be exceptionally poor in younger persons, being $50 \%$ and $76 \%$ in males and females < 30 years respectively increasing to $83 \%$ and $89 \%$ respectively in the group $\geq$ 50 years. The Cornell criterion specificity exceeded $90 \%$ at all ages in both sexes.

New diagnostic criteria for LVH must be age and sex dependent and ideally should undergo a thorough evaluation of specificity on a large database of apparently healthy individuals from both sexes aged 18 years and over before publication.
\end{abstract}

\section{Introduction}

Over the years, various electrocardiographic criteria for left ventricular hypertrophy (LVH) have been developed. The more widely used criteria are the Romhilt-Estes scoring system [1], the Sokolow-Lyon index [2] and the Cornell voltage criterion [3]. Sensitivity is generally low for all criteria but specificity varies from being acceptable to poor. For this reason, new criteria are sought. Due to the small numbers of patients used in studies on proposed criteria, the results obtained can sometimes be misleading.

A recent publication proposed a new criterion, namely the Peguero/Lo Presti ECG criterion for LVH [4]. The criterion was:

Max $\mathrm{S}$ wave amplitude $+\mathrm{S}$ wave amplitude in $\mathrm{V} 4>$ $2.8 \mathrm{mV}$ for males or $2.3 \mathrm{mV}$ for females.
In their paper, the authors reported an improved sensitivity index (62\%) compared to more commonly used criteria, as well as a high specificity (90\%). The population used for the study consisted of a small number of patients ( 47 males, 47 females, mean age $54 \pm 17$ years) with LVH proven by echocardiography and a similar number of patients in a control group (59 males, 63 females, mean age $68 \pm 15$ years). The authors listed the small sample size as a limitation of the study.

The aim of the present study was to assess the specificity of the new criterion on a larger study population of healthy individuals, with particular attention to the value of the index for different age groups within each gender group.

\section{Methods}

A dataset of apparently healthy Caucasian adults was used in the study. 1496 ECGs were analysed of which 859 were from males). The age range of the cohort was 18 to 82 years. The ECGs were analysed using version 28 of the University of Glasgow 12 Lead resting ECG analysis program (Glasgow program) [5]. The required wave measurements were extracted and the new Peguero/Lo Presti index was calculated. The SokolowLyon index (SV1 + RV5 amplitude) and the Cornell voltage (SV3 + RaVL amplitude) were also calculated.

ECGs were sub-divided into age groups for males and females, namely 18-29, 30-39, 40-49 and $\geq 50$ years. Counts were made of the number of cases that met the required thresholds for each index within each age and sex category within the Glasgow program, and resulting specificity was noted. Counts were also obtained of the number of cases that reported LVH using the Glasgow criteria. The Glasgow program uses a combination of the Romhilt-Estes criteria and the Cornell criteria.

\section{Results}

The specificity values are shown in Table 1 . The numbers of cases meeting the Peguero/Lo Presti criterion is high in the age groups 18-29 and 30-39 years. Of the 417 females under 40, $99(23.7 \%)$ met the criteria. Of the 448 males, 199 (44.4\%) met the criteria. 
Table 1. Specificity values for the Peguero/Lo Presti criteria, the Sokolow-Lyon criteria and the Cornell criteria by age and gender.

\begin{tabular}{|c|c|c|c|c|c|c|c|c|}
\hline \multirow{2}{*}{$\begin{array}{l}\text { Age } \\
\text { (years) }\end{array}$} & \multicolumn{2}{|l|}{$\mathrm{n}$} & \multicolumn{2}{|c|}{$\begin{array}{l}\text { Peguero- } \\
\text { Lo Presti }\end{array}$} & \multicolumn{2}{c|}{ Sokolow-Lyon } & \multicolumn{2}{c|}{ Cornell } \\
\cline { 2 - 9 } & $\mathrm{M}$ & $\mathrm{F}$ & $\mathrm{M}$ & $\mathrm{F}$ & $\mathrm{M}$ & $\mathrm{F}$ & $\mathrm{M}$ & $\mathrm{F}$ \\
\hline $18-29$ & 232 & 290 & $50 \%$ & $76 \%$ & $64 \%$ & $75 \%$ & $94 \%$ & $98 \%$ \\
$30-39$ & 216 & 127 & $61 \%$ & $78 \%$ & $77 \%$ & $94 \%$ & $98 \%$ & $94 \%$ \\
$40-49$ & 210 & 91 & $79 \%$ & $89 \%$ & $86 \%$ & $99 \%$ & $99 \%$ & $91 \%$ \\
$\geq 50$ & 201 & 129 & $83 \%$ & $89 \%$ & $91 \%$ & $95 \%$ & $99 \%$ & $94 \%$ \\
\hline
\end{tabular}

Overall, there were 400 ECGs that met the Peguero/Lo Presti criterion, 212 that met the Sokolow-Lyon criterion and 48 that met the Cornell criterion. There were four reports of LVH using the Glasgow Program that uses a combination of the Romhilt-Estes criteria and the Cornell criterion.

\section{Discusssion}

The published specificity for the Peguero/Lo Presti criterion was $89 \%$. It was evident from the study that while the Peguero/Lo Presti criterion resulted in high specificity for older age groups, the specificity was low for the younger age groups, namely under 40 years. The same is true for the Sokolow-Lyon index, in particular in the age group 18-29 years. The specificity for the Cornell Index is high for all categories of age and sex. An example of an ECG for which both the Peguero/Lo Presti criterion and the Sokolow-Lyon criterion are met, but which fails to meet the Cornell criterion is shown in Figure 1. The ECG is from an apparently healthy 34-year old male. With respect to the Peguero/Lo Presti criterion, there are prominent, but not abnormally large $S$ waves in $\mathrm{V} 2$ and V4, resulting in a combined amplitude of $3.49 \mathrm{mV}$ which exceeds the specified threshold for males of $2.8 \mathrm{mV}$. Similarly for Sokolow-Lyon, the sum of the $\mathrm{S}$ amplitude in lead V1 and R amplitude in V5 meet the criterion, while the sum of the S amplitude in V3 and $\mathrm{R}$ amplitude in aVL is $2.48 \mathrm{mV}$ which is insufficient to report LVH using the Cornell criterion for males.

Using the measurements from the 1496 examples, calculations were made to determine the thresholds that would be required to obtain $95 \%$ specificity using the Peguero/Lo Presti criterion. The thresholds for the age groups that were determined are given in Table 2.

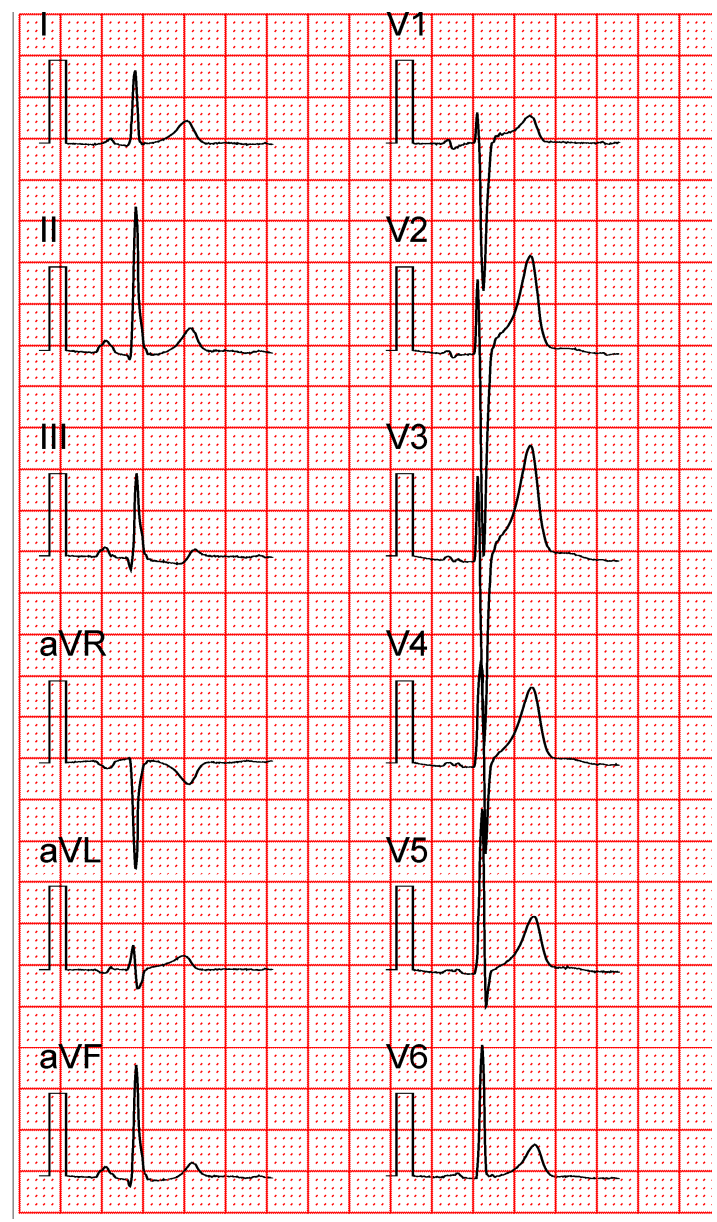

Figure 1. ECG of a 34 year old healthy male. The amplitudes meet the Peguero/Lo Presti criterion and the Sokolow-Lyon criterion but not the Cornell criterion or the Romhilt-Estes criteria. 
Table 2: Thresholds required to obtain $95 \%$ specificity using the Peguero/Lo Presti criterion.

\begin{tabular}{|l|l|l|}
\hline \multirow{2}{*}{$\begin{array}{l}\text { Age } \\
\text { (years) }\end{array}$} & \multicolumn{2}{|l|}{ Thresholds (mV) } \\
\cline { 2 - 3 } & Male & Female \\
\hline $18-29$ & 4.35 & 3.12 \\
$30-39$ & 4.03 & 3.21 \\
$40-49$ & 3.47 & 2.62 \\
$\geq 50$ & 3.50 & 2.75 \\
\hline
\end{tabular}

The Glasgow program uses a variation of the RomhiltEstes scoring system, combined with the Cornell criterion. An example of an ECG from a 47-year old male for which a false positive report of LVH was given by the Glasgow Program is shown in Figure 2.

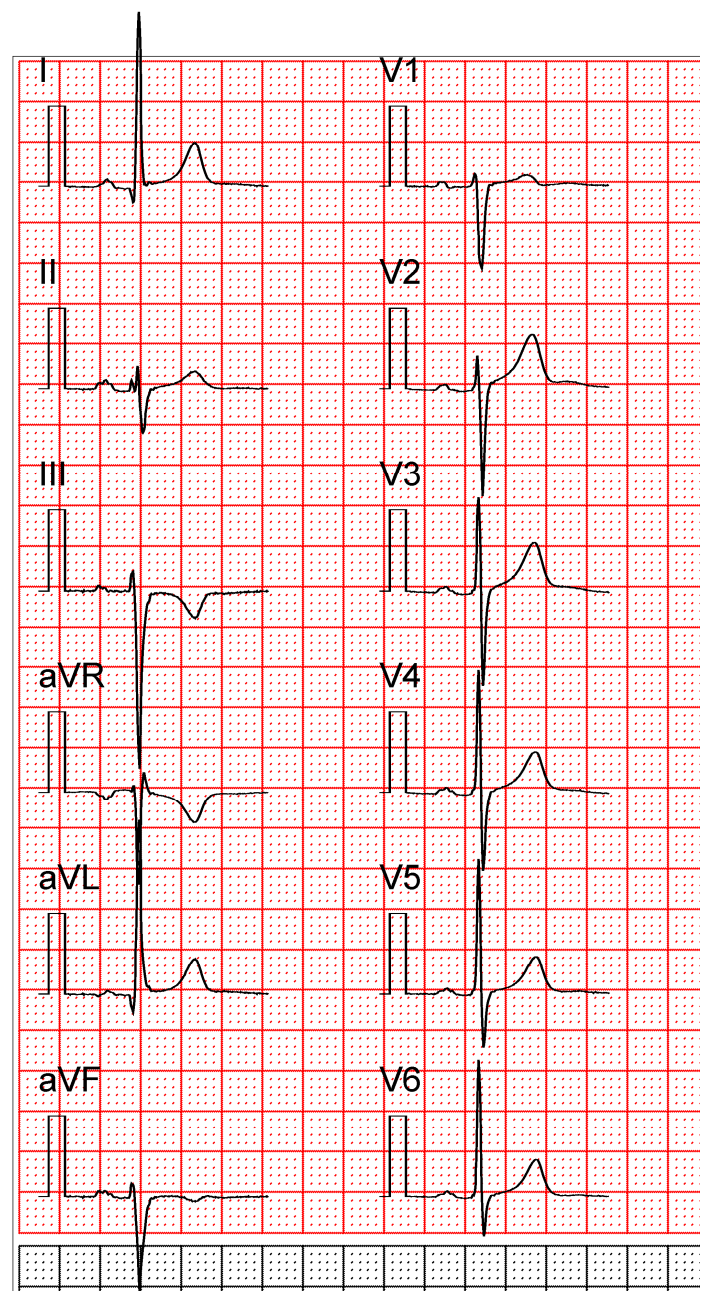

Figure 2. Waveforms for ECG of an apparently healthy 47-year old male. A diagnosis of left ventricular hypertrophy was reported by the Glasgow program.
In this case the $\mathrm{R}$ amplitudes is very high in the lead I and the $\mathrm{S}$ amplitude very deep in lead III. Using the Peguero/Lo Presti criterion or the Cornell criterion would also result in a false positive report of LVH for this ECG. The Sokolow-Lyon criteria were not met.

In their study, Peguero et al found that their proposed criterion had the best sensitivity score for identifying $\mathrm{LVH}$ for their test cohort. However, by using only $\mathrm{S}$ amplitudes in the criteria, cases of LVH which manifest as high $\mathrm{R}$ amplitudes as well as moderate to high $\mathrm{S}$ amplitudes in the limb leads may not be reported as LVH. Such an example is shown in figure 3 .

The presence of LVH in this 70 year-old man was determined on an increased indexed LV mass [6]. There is high $\mathrm{R}$ amplitude in leads I and aVL $(1.46 \mathrm{mV}$ and 1.86 $\mathrm{mV}$ respectively) as well as high $\mathrm{S}$ amplitude in leads III and $\mathrm{aVF}(2.28 \mathrm{mV}$ and $1.60 \mathrm{mV}$ respectively). The $\mathrm{S}$ amplitude in lead V4 was measured as $0.4 \mathrm{mV}$.

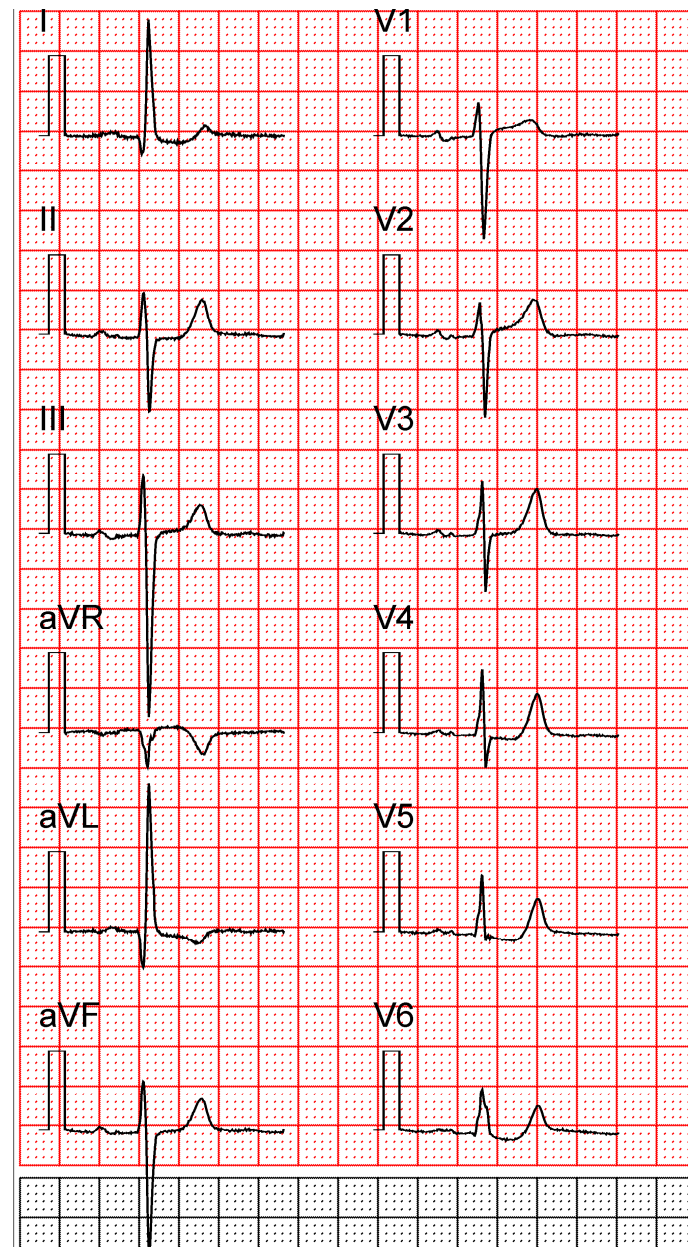

Figure 3. Waveforms for ECG of a 70-year old male, with confirmed LVH. The amplitudes fail to reach the the threshold for Peguero/Lo Presti criteria. 
The Romhilt-Estes point score system gave a high score but neither the Peguero/Lo Presti, Sokolow-Lyon nor Cornell voltage-based criteria were met. This case actually has a positive Lewis Index $(\mathrm{R} \mathrm{I}+\mathrm{S} \mathrm{III})-(\mathrm{R}$ III + $\mathrm{S} \mathrm{I})>1.7 \mathrm{mV}$ [7]. This index is highly specific but insensitive.

The voltage only criteria have the advantage of simplicity but have been found to have different levels of sensitivity. Automated algorithms allow combinations of criteria to be used and combining the scoring systems with voltage criteria, as in the Glasgow program, has been found to improve accuracy [7].

The low number of reports of LVH using the Glasgow program (4/1496) is due largely to the database having been used as a training set, which resulted in some modifications being made to the algorithm. For example, in order for LVH to be reported if the Cornell criteria are met, there must be no ST-T segment abnormalities.

In the Glasgow program, the thresholds used are age and sex dependent, and there are separate equations used for Caucasian and Oriental adults. It would be worthwhile considering race dependence when investigating new criteria for LVH given that blacks for example tend to have higher QRS amplitudes than Caucasians [8]. Indeed, black females appear to have an increasing Sokolow-Lyon index with advancing age [8].

\section{Conclusion}

It is well known that LVH criteria should be sex dependent and the new Peguero/Lo Presti criterion follows this method. New diagnostic criteria for $\mathrm{LVH}$ should also be age-dependent and, therefore, it is important to validate such criteria on all age groups. Ideally, new criteria for LVH should be thoroughly tested on all age groups. If possible, specificity should be evaluated on a large database of apparently healthy individuals from both sexes aged 18 years and over before publication. This is particularly true when younger individuals are being assessed for participation in sport [9].

\section{References}

[1] Romhilt DW, Estes Jr EH. A point-score system for the ECG diagnosis of left ventricular hypertrophy. Am Heart J 1968; 75: 752-8.

[2] Sokolow M, Lyon TP. The ventricular complex in left ventricular hypertrophy as obtained by unipolar precordial limb leads. Am Heart J 1949; 37: 161-86.

[3] Casale PN, Devereux RB, Kligfield P, et al. Electrocardiographic detection of left ventricular hypertrophy: development and prospective validation of improved criteria. J Am Coll Cardiol 1985; 6: 572-80.

[4] Peguero JG, Lo Presti S, Perez J, Issa O, Brenes JC, Tolentino A. Electrocardiographic Criteria for the Diagnosis of Left Ventricular Hypertrophy. J Am Coll Cardiol 2017; 69: 1694-1703.

[5] Macfarlane PW, Devine B, Clark E. The University of Glasgow (Uni-G) ECG analysis program. Computers in Cardiology 2005; 32: 451-4.

[6] Morrison I, Clark E, Macfarlane PW. Evaluation of the electrocardiographic criteria for left ventricular hypertrophy. Anadolu Kardiyol Derg 2007; 7: 159-163

[7] Macfarlane PW, Okin PM, Lawrie TDV, Milliken JA: Enlargement and Hypertrophy. In Macfarlane PW, van Oosterom A, Pahlm O, Kligfield P, Janse J, Camm J (Eds): Comprehensive Electrocardiology. 2nd Ed. London: Springer-Verlag, 2011: Vol 2 605-649.

[8] Macfarlane PW, Katibi IA, Hamde ST et al. Racial differences in the ECG - selected aspects. J Electrocardiol 2014;47:809-14.

[9] Froelicher V, Wagner G. The ECG and the pre-participation examination of young athletes. J Electrocardiol 2015; 48 : 281-282.

Address for correspondence.

Professor Peter W. Macfarlane

Institute of Health and Wellbeing

Electrocardiology Section, Level 1, New Lister Building,

Royal Infirmary

Glasgow

G31 2ER

Peter.Macfarlane@glasgow.ac.uk. 